\title{
No meio do caminho tinha um CAPSAD: centralidade e lógica assistencial da rede de atenção aos usuários de drogas
}

\author{
There was a CAPSad in the middle of the road: \\ care logic and centrality of the care network for drug users
}

Pedro Henrique Antunes da Costa ${ }^{1}$

Telmo Mota Ronzani ${ }^{1}$

Fernando Antonio Basile Colugnati ${ }^{1}$

${ }^{1}$ Programa de PósGraduação em Psicologia, Universidade Federal de Juiz de Fora. R. José Lourenço Kelmer s/n Campus Universitário, São Pedro. 36036-330 Juiz de Fora MG Brasil.

phantunes.costa@gmail.com

\begin{abstract}
By applying Social Network Analysis (ARS), this study seeks to evaluate the role of the Center for Psychosocial Care - Alcohol and Other Drugs (CAPSad) in the care network for drug users. It involves an exploratory, cross-sectional and quantitative approach of the Juiz de Fora city network in the state of Minas Gerais. One hundred and eighty-seven care services were identified in the city. The data gathering was made from a questionnaire with professionals of these instruments. The analysis took the cohesion and centrality metrics of the ARS into account as well as the creation of network sociograms. One centrality of the network was found in the CAPSad, in accordance with the policies and the care model advocated in the area, referred to here as "CAPSolization," The centralization in this instrument, still in insufficient number and with structural and workflow dynamics problems, leads to low resilience power of the network indicating the need for care logic modifications, still based on specialized and emergency care, to the detriment of territorial/community and ongoing prospects.
\end{abstract}

Key words Public health care, Substance-related disorders, Mental health, Substance abuse treatment centers, Public policies
Resumo $O$ presente estudo objetiva avaliar, através da Análise de Redes Sociais (ARS), o papel do Centro de Atenção Psicossocial - Álcool e outras Drogas (CAPSad) sobre a rede de atenção aos usuários de drogas. Trata-se de uma pesquisa exploratória, de corte transversal e abordagem quantitativa na rede de Juiz de Fora, Minas Gerais. Nela, foram identificados 187 serviços assistenciais no município. A coleta de dados foi feita a partir de questionário com profissionais destes dispositivos. A análise levou em consideração métricas de coesão e centralidade da ARS, bem como a confecção de sociogramas da rede. Constatou-se uma centralidade da rede no CAPSad, em conformidade com as politicas e o modelo assistencial preconizado na área, nomeada de "CAPSolização". A centralização neste dispositivo, ainda em número insuficiente e com problemas estruturais e na dinâmica de trabalho, influi para um baixo poder de resiliência da rede e indica a necessidade de modificação da lógica assistencial, ainda pautada pelo cuidado especializado, de urgência, em detrimento de perspectivas territorializadas/comunitárias e contínuas.

Palavras-chave Atenção à saúde, Transtornos relacionados ao uso de substâncias, Saúde mental, Centros de tratamento de abuso de substâncias, Políticas públicas 


\section{Introdução}

Atualmente, no Brasil, a assistência pública aos usuários de drogas é estruturada através da rede de atenção. Essa rede se constitui a partir de arranjos de governança, pressupondo tentativas de organização integrada de setores, serviços e profissionais para a oferta de um cuidado contínuo e de acordo com as necessidades da população, em detrimento de iniciativas isoladas e/ou sistemas fragmentados ${ }^{1,2}$.

Cabe ressaltar que este cenário atual é fruto de um processo histórico, que conformou uma série de avanços e conquistas no âmbito das políticas públicas e sociais, perpassando a saúde mental brasileira. Tal percurso tem seu momento de propulsão com a Reforma Psiquiátrica (RP), os Movimentos Sanitários e da Luta Antimanicomial nas décadas de 1970 e 1980, num conjunto de lutas e mobilizações sociais que culminaram em conquistas políticas, como a constituição do Sistema Único de Saúde (SUS). Acentua-se a partir da implementação do SUS um processo gradual de modificações na atenção às pessoas com transtornos mentais e usuários de drogas, visando uma reforma estrutural da concepção e modelo assistencial em saúde mental, com o início do fechamento dos hospitais psiquiátricos e a conformação de uma rede substitutiva, composta por serviços pautados nos direitos humanos e em direção à comunidade ${ }^{3}$.

Nesse contexto de desinstitucionalização, surgem no Brasil na década de 1980 dispositivos como o Centro de Atenção Psicossocial (CAPS) e o Núcleo de Atenção Psicossocial (NAPS), sendo os principais dispositivos substitutivos ao hospital psiquiátrico na oferta de uma atenção territorializada e contextualizada, por meio de propostas abertas. Partem do pressuposto de que a própria liberdade é terapêutica, devendo ser abordada como um dos elementos basilares da assistência ${ }^{3}$. Ao longo dos anos 1990 e início dos anos 2000, estes serviços passam por uma série de modificações e ampliações, com o estabelecimento dos tipos e modalidades de CAPS - como o CAPS Álcool e outras Drogas (CAPSad) -, seu funcionamento e as normas necessárias para cadastramento, obtendo também uma linha de financiamento específica do Ministério da Saúde $(\mathrm{MS})^{3}$.

No processo de aprofundamento da RP brasileira e consequente expansão dos serviços e rede substitutivos ao hospital psiquiátrico e sua lógica asilar/hospitalocêntrica, é sancionada a Lei 10.216, em 2001, e, em 2011, promulga-se a portaria que institui a Rede de Atenção Psicossocial (RAPS) ${ }^{4}$. A RAPS visa articular e ampliar os dispositivos assistenciais do SUS para pessoas com transtornos mentais e/ou usuários de drogas $^{4}$. São instituídos sete níveis de atenção, conformados pelos seguintes serviços: Unidades de Atenção Primária à Saúde (UAPS), equipes da Estratégia de Saúde da Família (ESF), Núcleos de Apoio à Saúde da Família (NASF), Consultórios na Rua (CRs), Centros de Convivência (CCs), leitos em Hospitais Gerais, Residências Terapêuticas (RTs), dentre outros ${ }^{4}$.

Um dos níveis da RAPS é a Atenção Psicossocial Especializada, constituída pelos CAPS. Articulados com outros pontos de atenção da rede, os CAPS são responsáveis por atender pessoas com transtornos mentais e/ou com necessidades devido ao uso de drogas, de maneira territorializada, por meio do Projeto Terapêutico Singular (PTS). São divididos em três tipos (I, II e III), de acordo com o tamanho do município e região de abrangência, estrutura e horário de funcionamento. Os CAPS III funcionam vinte e quatro horas todos os dias, possuindo também leitos de acolhimento. Além disso, os CAPS podem ser voltados especificamente para crianças e adolescentes (CAPSi) ou usuários de drogas (CAPSad).

Dessa forma, os CAPS adquirem papel fundamental na RAPS. Além de serem os principais dispositivos assistenciais especializados sobre a temática, também atuam como responsáveis pela sua articulação e fluxo de usuários, juntamente com a Atenção Básica ( $\mathrm{AB}$ ), e pelo suporte teórico-prático aos outros serviços, por meio do apoio matricial ${ }^{4}$. Especificamente na assistência aos usuários de drogas, os CAPSad que adquirem esse papel de gerenciador da rede, para além de apenas um serviço assistencial, sendo responsáveis pelo fluxo assistencial, através da referência, contrarreferência e cuidado compartilhado, assim como pelo matriciamento dos serviços não especializados, como as UAPS e equipes da $\mathrm{ESF}^{1,2,4}$.

Contudo, devido à própria complexidade da temática do uso de drogas, enquanto problema multifatorial e com uma série de determinantes sociais, entende-se que somente o setor saúde não é capaz de abranger essa amplitude. Os dispositivos do Sistema Único de Assistência Social (SUAS), como os Centros de Referência da Assistência Social (CRAS), Centros de Referência Especializados da Assistência Social (CREAS), Centros de Referência Especializados para População em Situação de Rua (CentroPOP) e serviços de acolhimento institucional (SAI) possuem grande 
importância na abordagem à temática, devendo ser incorporados à rede de atenção aos usuários de drogas ${ }^{5,6}$. Ademais, segundo as políticas na área ${ }^{1,2}$, não se deve desconsiderar os recursos comunitários, como os grupos de ajuda mútua (GAMs) e entidades socioassistenciais que atuam na assistência a usuários de drogas.

Dessa forma, pesquisas sobre o papel do CAPSad na rede de atenção ampliada e intersetorial aos usuários de drogas fazem-se necessárias. Revisões da literatura demonstram um crescimento na produção científica sobre os CAPS, mas com os estudos focando a avaliação de suas estruturas, propostas de atenção e resultados da assistência ${ }^{7,8}$. Contudo, Costa et al. $^{6}$ apontam a necessidade de se refletir sobre as funções dos CAPSad na rede, assim como suas práticas, visando maior contextualização das ações. Com as recentes mudanças nas políticas e a complexidade da temática das drogas, é necessário compreender o CAPSad enquanto um elemento da rede, sendo conformado por ela, atrelado ao entendimento do seu papel nessa conjuntura. Qual a posição do CAPSad na rede de atenção aos usuários de drogas? De que forma estabelece vínculos com outros serviços para o cuidado compartilhado?

Buscando responder a essas perguntas, o presente estudo objetiva avaliar, através da Análise de Redes Sociais (ARS), o papel do CAPSad na rede de atenção aos usuários de drogas. Dessa forma, é possível municiar alguns questionamentos colocados teórica ou hipoteticamente na literatura, por meio de dados empíricos concernentes à realidade, compreendendo limitações e potencialidades oriundas das políticas na área e perpassando o CAPSad, o CAPS, a RAPS e sua relação intra e intersetorial. Adicionalmente, almeja-se aprofundar as reflexões sobre redes como arranjos de governança nas políticas públicas sobre drogas, a partir de uma perspectiva abrangente, enfocando as dinâmicas de interação entre serviços, de modo a repercutir nas próprias políticas e práticas.

\section{Método}

\section{Aspectos gerais}

O presente estudo é um recorte de uma pesquisa guarda-chuva sobre a rede de atenção aos usuários de drogas, na qual será focalizado o papel do CAPSad na articulação desta rede e suas relações com outros dispositivos. Trata-se de uma pesquisa exploratória, de corte transversal e abor- dagem quantitativa, com a coleta de dados feita de maio de 2014 a fevereiro de 2015 na rede do município de Juiz de Fora. A cidade situa-se na região da Zona da Mata e Vertentes mineira. A população estimada é de 555.284 habitantes, numa extensão territorial de $1.436 \mathrm{~km}^{2}$, com densidade demográfica de 359,59 hab. $/ \mathrm{km}^{2}$. Seu Índice de Desenvolvimento Humano (IDH) é de $0,778^{9}$.

Seguindo as orientações das políticas da área $^{1,2,4}$, os serviços assistenciais abarcados pelo presente estudo poderiam ser governamentais ou não governamentais, com modalidades de tratamento diversificadas e constituintes dos seguintes âmbitos: (a) SUS, através da RAPS; (b) SUAS, organizando as ações da assistência e proteção social; e (c) iniciativas oriundas da própria comunidade sobre a temática, chamadas de recursos comunitários (GAMs, entidades socioassistenciais etc.), como forma complementar de ampliação e aprofundamento do escopo de cuidado, sem desconsiderar a responsabilidade e a centralidade do Estado e os dispositivos públicos na oferta assistencial.

\section{Procedimentos}

A utilização da ARS como ferramenta de análise ocorreu devido à possibilidade de identificação e mensuração das relações entre o CAPSad e os demais dispositivos da rede de atenção, de modo a entender a posição e o papel desempenhado por este serviço na rede. De acordo com Gomide e Grossetti ${ }^{10}$, a ARS caracteriza-se enquanto uma ferramenta que possibilita "identificar os tipos de relações existentes entre os atores, medindo de forma sistemática o comportamento relacional desses atores". Assim, a ARS adquire um caráter inovador nas ciências sociais e da saúde, ao focar nas relações entre nós (que podem ser pessoas, serviços etc.) de uma rede ou sistema, seja ela real ou virtual, facilitando a visualização de assimetrias, ambivalências e contradições, bem como a compreensão do papel das partes que compõem essa conjuntura e como essas relações influenciam a própria estrutura da rede $\mathrm{e}^{10-12}$.

A abordagem escolhida para a ARS foi a sociocêntrica, visando englobar todos os nós/serviços que faziam parte da rede e suas ligações (laços) uns com os outros ${ }^{13}$. Para isso, a estratégia de coleta dos dados para a ARS foi delineada a partir de um survey por saturação (saturation survey), método comumente utilizado para redes sociocêntricas ${ }^{14}$. A rede foi mapeada por completo, com a amostragem do tipo censo, considerando as seguintes etapas: (a) levantamento preliminar 
da rede através de ferramentas, documentos da gestão municipal e bases de dados ministeriais públicas; (b) contato com secretarias gestoras municipais e entidades de participação social sobre a temática; e (c) método de bola de neve $(\text { snowball })^{15}$ com profissionais da rede.

A partir desse processo, foram identificados 187 serviços assistenciais, que compunham a rede de atenção aos usuários de drogas no município, com os participantes da pesquisa sendo profissionais destes dispositivos e fornecendo os dados relacionais de suas respectivas instituições. Para a escolha destes participantes, entrou-se em contato com os serviços e seus coordenadores/ gestores, perguntando sobre os profissionais com maior conhecimento sobre a instituição e sua relação com a rede. Aqueles indicados foram convidados a participar do estudo.

Os dados foram coletados através de questionários aplicados por pesquisadores treinados. Os questionários foram construídos na plataforma Formhub (https://formhub.org/), que é um serviço da internet gratuito para a construção e gestão de surveys online e bancos de dados. As entrevistas foram feitas com o auxílio de tablets, com as respostas assinaladas nesses dispositivos móveis. Visando a adequação dos instrumentos, conteúdo, linguagem e a própria forma de aplicação, foram realizados 15 pré-testes.

\section{Instrumento}

Para o presente estudo, o foco será nos resultados provenientes do Questionário de Relacionamentos $(\mathrm{QR})$, voltado para a análise de padrões, estruturas e a tipologia das interações estabelecidas entre o CAPSad e os elementos da rede. Nele, os participantes responderam à seguinte pergunta: No último ano, com quais dispositivos (listados no menu) o seu serviço teve algum tipo de relação/atividade em conjunto referente à atenção aos usuários de drogas? Todas as relações foram do tipo unidirecional, isto é, o serviço A poderia ter relação com o $\mathrm{B}$, mas isso não significa que $\mathrm{B}$ tenha automaticamente relação com A. Como suporte para as respostas do QR, foi confeccionado e entregue para cada respondente um menu com os serviços mapeados da rede, para que pudessem consultar durante a aplicação dos questionários, minimizando esquecimentos.

\section{Análise dos dados}

A análise dos dados foi realizada com o auxílio do software $R$ Project for statistical compu- ting ${ }^{16}$. Após o preenchimento dos questionários, os dados foram exportados, em forma de matrizes de adjacência, do repositório no Formhub para o software $R$, de onde foram extraídas todas as métricas da ARS. Posteriormente, foram gerados sociogramas das redes a partir do pacote IGraph ${ }^{17}$, com os nós tendo os seus tamanhos ajustados de acordo com suas Centralidades de Grau (CG). Logo, quanto maior o número de ligações feitas por um dispositivo, maior ele se encontra nos sociogramas.

A análise das relações dessa rede compreende a identificação das unidades mais conectadas, as centrais e as intermediárias, a formação de subgrupos e as interconexões entre as unidades. As métricas fornecidas pela ARS procuram quantificar estas características, sendo as utilizadas descritas abaixo:

- Métricas de Centralidade: 1) Centralidade de Grau (CG), que é a soma de todas as ligações feitas por um determinado ator, indicando níveis de atividade ou popularidade; 2) Centralidade de Intermediação (CI), a medição do quanto um nó pode controlar/mediar o fluxo de informações, dada sua posição na rede; e 3) Centralidade de Proximidade (CP), que mede quão próximo um nó está em relação aos outros, sendo uma medida de alcance da informação a um nó específico.

- Métricas de Coesão: 1) Grau, indicando a média e mediana da somatória de relações dos serviços; 2) Densidade, que é a proporção entre o número de vínculos observado e o total possível na rede; 3) Distância média, a medida do número médio de laços que separam dois nós; 4) Cliques, o número de agrupamentos entre três ou mais nós conectados, que formam subgrupos dentro da rede; e 5) Transitividade: a probabilidade de que um ator se conecte a atores adjacentes, devido às conexões já existentes.

Posteriormente, de modo a aprofundar a compreensão sobre o papel do CAPSad, ele foi retirado da rede, com recálculo das métricas de coesão da rede e de centralidade dos serviços, para que fosse possível realizar uma comparação posterior dessas mesmas métricas com e sem o CAPSad. Por fim, a partir dos resultados encontrados, foi realizada uma análise comparativa entre o modelo de organização da rede de atenção aos usuários de drogas apregoado pelas políticas da área e o encontrado no município.

\section{Aspectos éticos}

O presente estudo foi aprovado pelo Comitê de Ética da Universidade Federal de Juiz de Fora, 
com os participantes assinando o Termo de Consentimento Livre e Esclarecido.

\section{Resultados}

De acordo com a Tabela 1, o CAPSad é o dispositivo da rede com as maiores métricas de centralidade (CG, CI, CP). Tal cenário também é ilustrado na Figura 1, que demonstra os dispositivos (nós) e suas inter-relações. Nesse sentido, observa-se uma centralização da rede no CAPSad, tomado como dispositivo regulador do fluxo assistencial ao usuário de drogas e do processo de trabalho.

Quando apresentados ao modelo pressuposto pelas políticas na área, conforme ilustrado na Figura 2, comparando-o, inclusive com o sociograma (Figura 1) e as métricas de centralidade da ARS (Tabela 1), observa-se uma similaridade com a estruturação da rede de atenção encontra- da no município. A única ressalva com relação à Figura 2 é que ela representa uma rede de saúde mental geral, havendo a necessidade de substituir o CAPS pelo CAPSad como elemento central na rede de atenção aos usuários de drogas.

Essa centralização no CAPSad fica mais evidente quando ele é retirado da rede e, a partir disso, novas métricas de coesão são extraídas e analisadas. A Tabela 2 apresenta esses dados da rede geral com e sem o CAPSad. Com a retirada deste dispositivo, constatou-se uma diminuição de 211 laços, o que corresponde a uma redução de 13,3\% das relações estabelecidas pela rede. Ademais, houve um decréscimo nos valores de todas as métricas, com exceção da distância média, o que era esperado, afinal as maiores distâncias indicam que sem o CAPSad, a trajetória na rede tende a ser maior e, portanto, mais demorada.

Tais dados indicam uma rede com alta dependência desse serviço e, consequentemente, uma rede com baixa capacidade de resiliência,

Tabela 1. Métricas de Centralidade dos dispositivos da rede de acordo com suas tipologias.

\begin{tabular}{|c|c|c|c|c|c|c|}
\hline \multirow{3}{*}{ Serviços } & \multirow{3}{*}{$\mathbf{N}$} & \multicolumn{5}{|c|}{ Métricas de Centralidade } \\
\hline & & \multicolumn{3}{|c|}{$\begin{array}{l}\text { Grau } \\
(\mathrm{CG})\end{array}$} & \multirow{2}{*}{$\begin{array}{c}\text { Intermediação } \\
(\mathrm{CI})\end{array}$} & \multirow{2}{*}{$\begin{array}{c}\text { Proximidade } \\
(\mathrm{CP})\end{array}$} \\
\hline & & $\begin{array}{l}\text { Entrada } \\
\qquad \mathrm{M}\end{array}$ & $\begin{array}{c}\text { Saída } \\
\text { M }\end{array}$ & $\begin{array}{c}\text { Geral } \\
\mathrm{M}\end{array}$ & & \\
\hline CAPSad & 1 & 106 & 105 & 211 & 1 & 0,003968 \\
\hline HPS & 1 & 70 & 89 & 159 & 0,412853 & 0,003774 \\
\hline Ambulatório em saúde mental & 1 & 38 & 64 & 102 & 0,220140 & 0,003175 \\
\hline SAMU & 1 & 39 & 20 & 59 & 0,032856 & 0,002849 \\
\hline CREAS & 3 & 21,7 & 35,7 & 57,4 & 0,031724 & 0,002854 \\
\hline $\begin{array}{l}\text { Hospital Geral com leitos em álcool e outras } \\
\text { drogas }\end{array}$ & 1 & 27 & 18 & 45 & 0,107471 & 0,002976 \\
\hline CentroPOP & 1 & 26 & 16 & 42 & 0,019838 & 0,002717 \\
\hline Ambulatório sobre tabagismo & 1 & 14 & 27 & 41 & 0,014446 & 0,002618 \\
\hline CAPS gerais & 3 & 24,7 & 15,3 & 40 & 0,024841 & 0,002677 \\
\hline CRAS & 9 & 19,1 & 18,1 & 37,2 & 0,029329 & 0,0027 \\
\hline Hospital Psiquiátrico & 1 & 14 & 21 & 35 & 0,031737 & 0,002653 \\
\hline Serviços de Acolhimento Institucional & 3 & 14 & 17,7 & 31,7 & 0,022821 & 0,002552 \\
\hline Consultórios na Rua & 2 & 7,5 & 23,5 & 31 & 0,017176 & 0,002677 \\
\hline CAPSi & 1 & 21 & 9 & 30 & 0,004311 & 0,002653 \\
\hline Entidades Socioassistenciais & 11 & 8,4 & 12,4 & 20,8 & 0,018275 & 0,002478 \\
\hline Ambulatório em álcool e outras drogas & 1 & 9 & 9 & 18 & 0,020618 & 0,002532 \\
\hline Centro de Convivência & 1 & 14 & 2 & 16 & 0,031189 & 0,0025 \\
\hline Clínicas e Comunidades Terapêuticas & 19 & 5,8 & 8,6 & 14,6 & 0,013326 & 0,002357 \\
\hline UAPS Urbanas & 48 & 6,7 & 5 & 11,7 & 0,005326 & 0,002477 \\
\hline Residências Terapêuticas & 12 & 3,8 & 5,1 & 8,9 & 0,000536 & 0,002324 \\
\hline Grupos de Ajuda Mútua & 46 & 4 & 3,3 & 7,3 & 0,006577 & 0,002121 \\
\hline UAPS Rurais & 19 & 4,1 & 1,7 & 5,8 & 0,000123 & 0,002411 \\
\hline Hospital Judiciário & 1 & 0 & 0 & 0 & 0 & 0,002288 \\
\hline
\end{tabular}

Fonte: Dos Autores. 

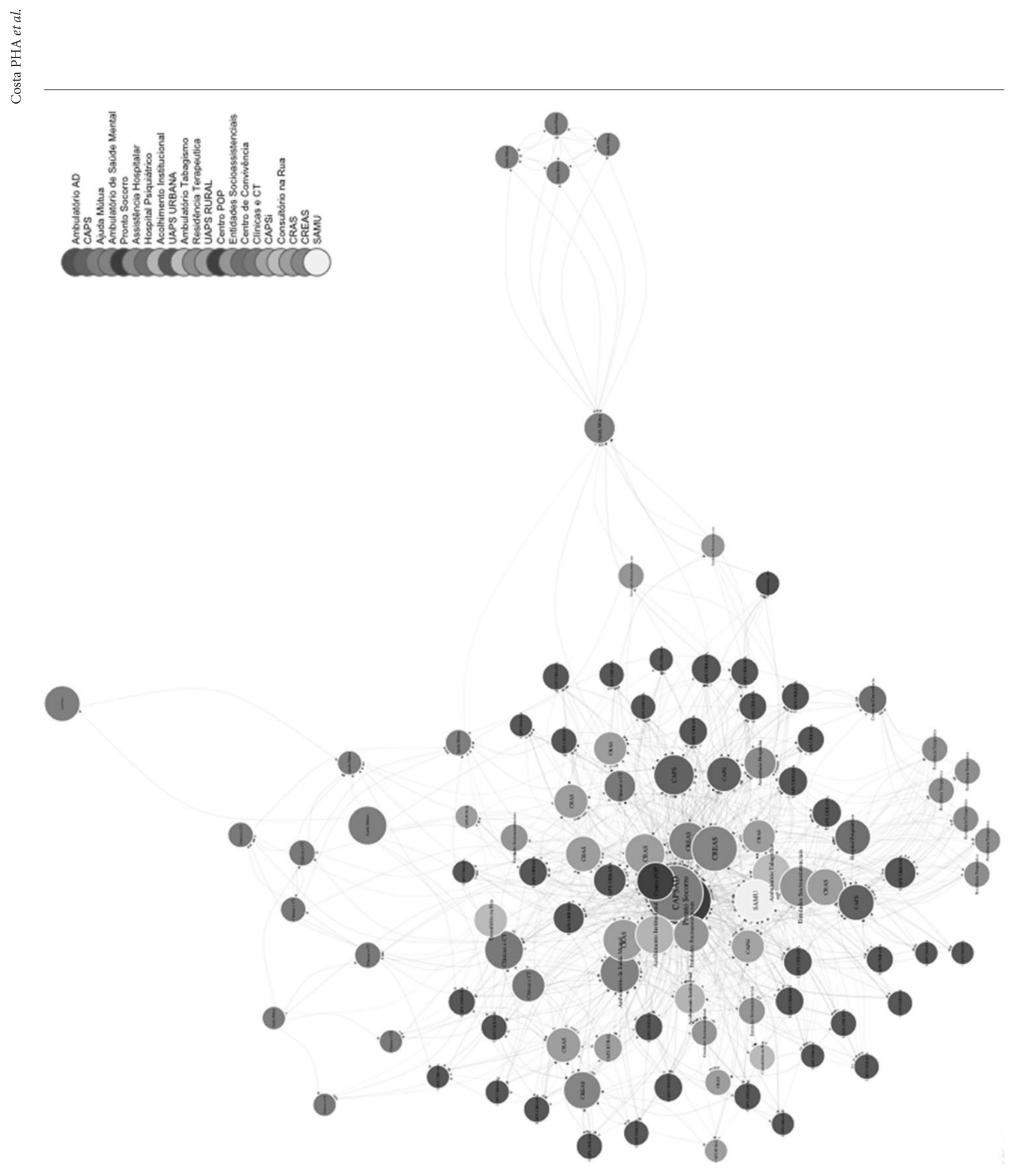

Figura 1. Sociograma da rede de atenção.

Fonte: Dos Autores.

pois, com a retirada do seu principal dispositivo, cujas métricas de centralidade são as mais elevadas e dissonantes, uma parcela significativa das relações estabelecidas na rede também é ex- cluída - dada a sua já baixa densidade -, assim como suas métricas de coesão são diminuídas. Ademais, os outros dispositivos especializados (principalmente os ambulatórios) e os de urgên- 


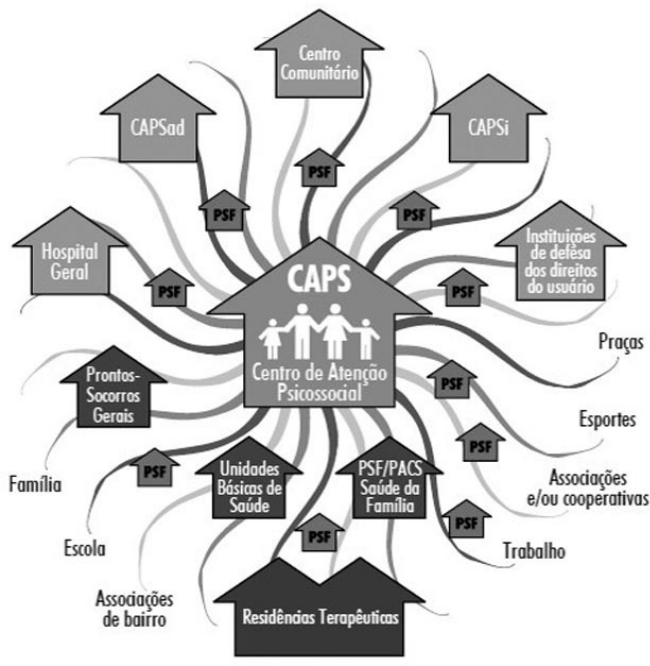

Figura 2. Rede de Atenção à Saúde Mental.

Fonte: TCU (2012), adaptado de Ministério da Saúde (2004).

Tabela 2. Métricas de coesão da rede com e sem oCAPSad.

\begin{tabular}{lr}
\hline \multicolumn{2}{c}{ Com o CAPSad } \\
\hline Número de Nós & 187 \\
Número de Laços & 1580 \\
Grau - Média & 16,9 \\
Grau - Mediana & 10,5 \\
Densidade & $4,54 \%$ \\
Distância Média & 2,53 \\
No de Cliques & 11 \\
Transitividade & 0,251 \\
\hline & \\
\hline Número de Nós & 186 \\
Número de Laços & 1369 \\
Grau - Média & 14,72 \\
Grau - Mediana & 7 \\
Densidade & $3,98 \%$ \\
Distância Média & 2,75 \\
No de Cliques & 10 \\
Transitividade & 0,245 \\
\hline
\end{tabular}

Fonte: Dos Autores.

cia/emergência (HPS e SAMU) com os maiores valores médios das métricas de centralidade, indicam uma tendência de centralização e dependência da rede nas instituições referentes a estes níveis de atenção, em detrimento de dispositivos generalistas e de base comunitária/territorial. Tal constatação pode ser mais bem visualizada na Figura 3, que representa o sociograma da rede sem o CAPSad. Nela, é possível observar o maior tamanho destes dispositivos especializados e/ou de urgência/emergência, devido às suas maiores métricas de centralidade.

\section{Discussão}

Antes de adentrar na discussão sobre os modelos assistenciais, cabe ressaltar a relevância histórica dos CAPS nas políticas e redes de saúde mental, atenção psicossocial e aos usuários de drogas. A relevância e a centralidade desse dispositivo na rede verificada pelo presente estudo é fomentada desde os primórdios da RP brasileira e perpassa os demais contextos e localidades do país. Uma centralidade que vai sendo reforçada no decorrer dos anos 1990 e início dos anos 2000, com o aprofundamento da Reforma e do processo de expansão dos serviços substitutivos, culminando com a promulgação da RAPS em 20114. O próprio crescimento na produção científica sobre os CAPS apontado por revisões da literatura nacional, bem como seus resultados no processo de cuidado ${ }^{7,8}$, também demonstram o protagonismo deste dispositivo nas políticas e redes da saúde mental e aos usuários de álcool e de outras drogas no Brasil.

Tal centralização será chamada aqui de "CAPSolização" da rede ou rede "CAPSolizada", fazendo alusão à ideia do CAPSad como um sol, com outros dispositivos orbitando em torno dele, isto é, se relacionando por meio dele. Salienta-se que Amarante $^{18}$ já havia anteriormente utilizado o termo "Capsização" para se referir à centralidade do CAPS na política de saúde mental, alertando sobre o caráter contraditório desta centralização frente à própria ideia de uma rede substitutiva e comunitária.

Então, deve-se compreender que os postulados a seguir não se tratam de críticas vazias e tentativas de deslegitimação a esse dispositivo, bem como os modelos de organização da rede propostos pelas políticas, muito menos de considerá-los descolados de seus contextos, como se fossem também imunes ao processo histórico de consolidação de práticas na área. Inclusive, o título do artigo, não se trata de uma tentativa de descaracterização do serviço, mas possui caráter meramente ilustrativo do quanto a lógica de "CAPSolização" (por isso o CAPS no meio do caminho; no centro do fluxo), encontra-se presente 


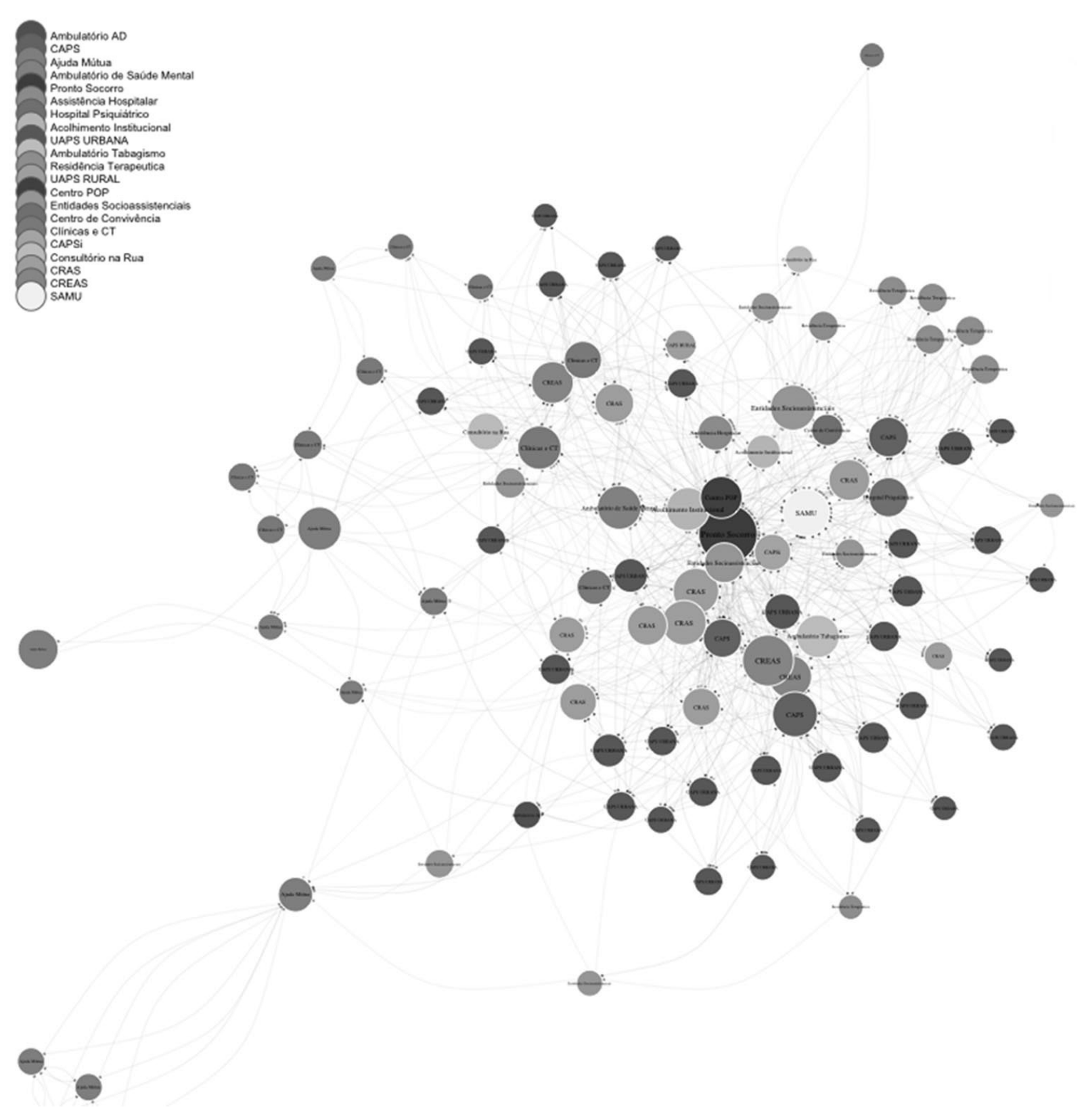

Figura 3. Sociograma da rede sem o CAPSad.

Fonte: Dos Autores.

tanto na rede real/concreta, do cotidiano, quanto na ideal, aquela propagada pelas políticas. Pretende-se, assim, somente elucidar algumas reflexões a partir da dialética ideal-real, circunscrita no jogo das políticas e práticas da área, ancorando-se em dados da realidade concreta, ao invés de considera-las como instâncias fragmentadas e dicotômicas.
Levando em consideração essas ponderações, os achados aqui discutidos correspondem ao local estudado, mas com algumas generalizações permitidas - guardadas as devidas proporções ao contexto nacional, sobretudo, a partir do diálogo estabelecido com as políticas e os resultados de avaliações e pesquisas na área. Por exemplo, quando comparadas a estruturação da rede de 
atenção do município e o modelo definido pelas políticas (Figura 2), constata-se uma semelhança entre ambos, ao menos no que se refere à "CAPSolização" da rede, fazendo com que alguns pontos sejam problematizados. O primeiro, é que esse cenário chamado de "CAPSolização" é recorrente na literatura da área ${ }^{6-8,19,20}$, o que demonstra o seu caráter nacional, como um caminho desenvolvido pelas políticas na saúde mental e álcool e outras drogas, não sendo apenas restrito ao município estudado, possibilitando, assim, reflexões mais amplas.

Em segundo lugar, constata-se ainda uma insuficiência na quantidade destes dispositivos no país, mesmo com a expansão observada nos últimos anos. Segundo a Sala de Gestão Estratégia do MS (SAGE) ${ }^{21}$, existem 406 CAPSad em funcionamento no Brasil, com grande concentração nas regiões Sul e Sudeste e sendo apenas 90 do tipo III (com funcionamento $24 \mathrm{~h}$, todos os dias). $\mathrm{Na}$ própria rede estudada há somente um CAPSad do tipo III, com abrangência que, inclusive, vai além do próprio município, abarcando cidades vizinhas da macrorregião de saúde. Sendo assim, no contexto estudado, surgem as seguintes indagações: Será que um dispositivo apenas seria capaz de prestar assistência aos casos existentes, bem como organizar o fluxo de cuidado da rede e gerência da clínica, ainda mais quando se considera uma rede com essa extensão de dispositivos? Será esse número suficiente no município para realizar o apoio matricial ou cuidado compartilhado com todas as 67 UAPS, por exemplo? E com os outros dispositivos e níveis de atenção? Ao se tratar de um panorama nacional, e não uma exclusividade local, tais questionamentos podem ser ampliados, guardadas as particularidades dos contextos.

Observa-se, portanto, um dispositivo sobrecarregado, ao menos em termos das demandas de relações com os outros dispositivos da rede, apontando, num primeiro momento para a necessidade implantação de mais CAPSad no município. Se utilizarmos o parâmetro de cobertura em saúde mental estabelecido pelo MS, que considera adequada a existência de um CAPS para cada 100 mil habitantes, a cobertura dos CAPS em Juiz de Fora seria de 0,95, é avaliada como muito boa (índices acima de 0,70). Para fins comparativos, tomando como base os dados da $\mathrm{SAGE}^{21}$ referente aos números de CAPS no país, em Minas Gerais o indicador médio também é de 0,95 e, no Brasil, 0,86, sendo que em 2002 era de 0,21. Entretanto, a própria centralidade do CAPSad na rede, associada ao número de rela- ções que se demanda dele, faz com que avaliemos criticamente este parâmetro de cobertura do MS e sua adequabilidade, em especial, para abarcar as especificidades geográficas e carências regionais/ locais ${ }^{22}$. Como exemplo, encontra-se o estudo sobre a variabilidade de cobertura dos CAPS no Rio Grande do Sul (o terceiro estado com maior índice de cobertura do país), mas que constatou sete regiões com cobertura insuficiente/inadequada, representando $49 \%$ da população do estado ${ }^{22}$. Ademais, questiona-se a utilização indiferenciada deste parâmetro para saúde mental em geral e álcool e outras drogas, desconsiderando as especificidades e necessidades de cada temática e área.

Tais questionamentos tornam-se ainda mais intricados quando comparados os números de CAPSad, seus níveis de cobertura no país e os investimentos públicos que lhes são dispendidos com os de outras instituições não governamentais, como as CTs, por exemplo ${ }^{23-25}$. Dessa forma, observa-se um cenário contraditório, onde apesar de mencionado como o principal dispositivo da rede de atenção aos usuários de drogas, e ocupando, de fato, uma posição central na rede, o CAPSad é, ao mesmo tempo, tomado de forma marginal pelo Estado brasileiro, com repasse de verbas aquém das condições necessárias de funcionamento e cumprimento de suas atribuições.

Aliam-se a esse cenário de insuficiências, os entraves na dinâmica de trabalho destes serviços, que, apesar de não serem alvos do presente estudo, influenciam diretamente no processo organizativo dos CAPSad, sendo constantemente retratados na literatura da área, como: carências na formação profissional, atrelada ao modelo biomédico e visões moralizantes, estigmatizantes e naturalizantes ${ }^{26-28}$; sobrecarga de trabalho aliada à escassez de recursos e estrutura deficitária ${ }^{27,29-31}$, dentre outros. Ademais, são inseridas nesse contexto as deficiências referentes a outros dispositivos, níveis de atenção e setores ${ }^{5,6,19,29}$, o que complexifica ainda mais a organização da rede de atenção através dessa lógica "CAPSolizada" e denota a importância de uma maior horizontalidade, o que não nega a necessidade uma hierarquização e institucionalização de fluxos de cuidado, vide a própria configuração dos níveis de atenção.

Os outros obstáculos dizem respeito à própria centralidade do CAPSad e aos contratempos provenientes, fazendo com que os seguintes questionamentos também se tornem pertinentes: A centralidade nesse dispositivo não contradiz o próprio conceito de rede de atenção, assim como a lógica de cuidado comunitário e territorializa- 
do oriundos da RP? Não estaríamos, portanto, reforçando um modelo contraditório ao postulado pela RP, onde no lugar do hospital psiquiátrico são inseridos outros dispositivos, mesmo que mais humanos, como os CAPS e CAPSad? Enfim, aponta-se a necessidade de se repensar a lógica que direciona o papel e a função destes dispositivos na rede e a própria organização destes arranjos organizativos. Afinal, como foi possível observar, os dispositivos com maiores métricas de centralidade depois do CAPSad foram serviços também especializados e de urgência/emergência, sendo que com a retirada do CAPSad nas análises, estes mesmos dispositivos passaram a "ocupar" sua posição em termos de centralidade na rede.

Entende-se que a RP e suas conquistas históricas compreendem não apenas uma troca do hospital psiquiátrico por outros serviços, como os CAPS e as demais estratégias substitutivas e extra-hospitalares. Vasconcelos ${ }^{32}$, por exemplo, destaca que essa centralidade na implantação dos CAPS, fez com que outros dispositivos e pontos da rede, como os leitos em hospitais gerais, fossem desconsiderados ou não tivessem o dispêndio devido de atenção e fomento de acordo com suas relevâncias: "[...] na expectativa de chegar imediatamente ao nosso objetivo estratégico, ou seja, no CAPS III, reduzimos, congelamos ou deixamos sucatear nossa retaguarda de leitos de atenção integral". Por mais que a mera substituição destes serviços já configurasse um considerável avanço, a RP pressupôs uma mudança radical e ruptura na forma como se concebe a saúde mental e o uso de drogas, bem como os modelos assistenciais e as instituições que as abordam e, consequentemente, em como as pessoas e seus contextos de vida deveriam ser compreendidos e tratados.

Por mais que o decreto da RAPS coloque que a ordenação do cuidado estará sob a responsabilidade dos CAPSs ou da $\mathrm{AB}$, as políticas de saúde mental do $\mathrm{MS}^{1,3}$ vão nessa direção de centralidade organizativa da rede e de gestão da clínica nos CAPS e CAPSad. Dessa forma, o cenário encontrado no município mostra uma replicação de orientações provenientes de cima para baixo, ou seja, do MS. Assim, trata-se de um problema, cuja origem consta desde a sua formulação, demonstrando que uma série de possíveis entraves que permeiam o cotidiano das ações advém dos próprios instrumentos norteadores, como, nesse caso, das políticas em si.

Além disso, tal panorama reforça a necessidade da configuração e institucionalização de flu- xos assistenciais, por meio de linhas-guia de cuidado, perpassado por uma hierarquização não tão rígida entre os dispositivos e níveis de atenção e com foco na $A B$, visando reverter ou, pelo menos, minimizar essa centralização no CAPSad, nos demais serviços especializados e/ou nos de urgência/emergência. Portanto, é necessária uma maior consideração da $\mathrm{AB}$ na gerência da clínica e ordenação dos fluxos assistenciais. Entretanto, não pretendemos uma mera responsabilização deste nível de atenção e seus respectivos dispositivos, já sobrecarregados, sem as necessárias modificações: maior dispêndio orçamentário, ampliações estruturais, melhores condições de trabalho etc. ${ }^{6,33}$.

Enfatizar a institucionalização de fluxos e linhas-guia não significa desconsiderar as singularidades de cada caso, abarcadas por meio dos PTS, mas somente que elas devem ser pensadas em conjunto com o que se tem de estabelecido, isto é, as possibilidades e caminhos pré-definidos na rede. Sem isso, corre-se o risco de uma centralidade e demanda cada vez maior nos já sobrecarregados serviços especializados ${ }^{34,35}$, acarretando em uma cronificação do usuário na rede ${ }^{36,37}$, assim como imobilismos por parte dos profissionais ${ }^{29}$, reforçados por problemas estruturais e esforços contínuos de "invenções de roda" demandados por cada novo caso que lhes é apresentado. Os próprios fluxos, além de não serem tomados de forma engessada, como receituários ou panaceias, devem ser formulados a partir das realidades locais. Através dessas estratégias, até mesmo a elaboração de novas saídas e possibilidades que fujam do instituído fica facilitada, a partir do momento em que já se tem um conjunto de caminhos e fluxos formalizados.

De modo geral, o que se observou no estudo foi que, apesar da retirada do CAPSad da rede resultar numa perda de cerca de $13 \%$ das relações estabelecidas, assim como na redução nos valores das métricas de coesão, essa diminuição não ocasionou uma desarticulação da rede, o que poderia ser visto com um aumento significativo nos números de cliques. Entretanto, isso pode ser explicado pelas densidades observadas nas redes, que encontram-se entre $3-5 \%$. Por mais que se tenha uma centralidade no CAPSad, a "baixa densidade" dessa rede faz com que o cenário não seja demasiadamente modificado. $\mathrm{Ou}$ seja, a retirada do CAPSad da rede não resulta em sua dissipação, pelo fato dela já se apresentar incipientemente articulada. Cabe ressaltar que utiliza-se "baixa densidade" mais na falta de uma outra adjetivação, devido à insuficiência de pa- 
râmetros que estipulem o que é uma rede com baixa, média e alta densidade, bem como estudos avaliativos prévios que possibilitem comparação. Além disso, devido à heterogeneidade da rede, alguns serviços possuem particularidades que dificultam uma valoração dessa densidade, ao impactarem nas relações que estabelecem (ou deixam de estabelecer). Isso pode ser visto, por exemplo, com os GAMs que, devido às suas filosofias, constituem cliques praticamente só com outros GAMs (Figura 3). Portanto, a não dissipação da rede não significa uma refutação da tese de "CAPSolização", apenas que, em grande parte, temos um conjunto de serviços que não se encontram articulados, fazendo com que pensemos se temos uma rede ou um emaranhado. Como consequência óbvia, aponta-se para a necessidade de uma maior integração entre os dispositivos, por meio da conformação de fluxos de cuidado.

Com relação às limitações da pesquisa, são destacadas: possíveis desconsiderações de serviços, especialmente os não governamentais, e presumíveis vieses de resposta dos participantes. Não obstante, existe uma insuficiência de parâmetros nacionais acerca das necessidades e da cobertura assistencial referente às pessoas com problemas relacionados ao uso de drogas, que dificultam o aprofundamento de algumas reflexões sinalizadas no presente trabalho. As especificidades socioculturais do contexto brasileiro e o próprio modelo assistencial na área de saúde mental e álcool e outras drogas aqui construído e implementado, por mais que influenciado por outros contextos, também obstaculizam e, em alguns casos, até mesmo impossibilitam a realização de comparações com outros países.

\section{Considerações finais}

O presente estudo constatou uma centralidade da rede de atenção aos usuários de drogas no CAPSad, em conformidade com as políticas e o modelo assistencial preconizado. A centralização neste dispositivo, ainda em número insuficiente e perpassado por uma série de problemas estruturais e na dinâmica de trabalho, além de influir para um baixo poder de resiliência da rede, também indica a necessidade de modificação da lógica assistencial na área, ainda pautada pelo cuidado especializado e/ou pontual, de urgência/ emergência, em detrimento de perspectivas territorializadas/comunitárias por meio de um cuidado contínuo. Dessa forma, o problema não reside no CAPSad, muito menos na proposta de rede em si, mas na racionalidade que ainda persiste na área de centralização das propostas assistenciais em dispositivos especializados, aliada às insuficiências na formulação e implantação das políticas, mesmo num cenário de inúmeros avanços provenientes da RP brasileira.

Agregando todo o montante de dados, juntamente com as reflexões e discussões suscitadas, aponta-se para a necessidade dos estudos futuros analisarem e compreenderem o CAPSad, bem como outros dispositivos, não só através de seus componentes estruturais, seus processos e dinâmicas de trabalho e resultados, mas como eles são influenciados pela estruturação da rede e os relacionamentos estabelecidos com outros serviços. Isso significa localizá-lo enquanto parte dessa rede, sendo constituído por ela e a conformando, de modo a entender também qual é o papel desempenhado por ele nesse panorama. Acredita-se que, assim, seja possível abarcá-lo da forma contextualizada que pressupõem as políticas e o aparato normativo-legal da área, bem como a própria história, para que, consequentemente, sejam visualizados e apontados direcionamentos em vistas à sua potencialização, como importante estratégia na área e materialização de uma série de avanços.

\section{Colaboradores}

PHA Costa, TM Ronzani e FAB Colugnati participaram de todas as etapas de construção e condução da pesquisa e escrita do artigo.

\section{Agradecimentos}

À Amata Medeiros, Bárbara Loures, Juliana Salgado, Mayara Custódio, Taynara Formagini e Wanderson Duarte pela parceria na condução da pesquisa. 


\section{Referências}

1. Brasil. Ministério da Saúde (MS). A Política do Ministério da Saúde para Atenção Integral a Usuários de Álcool e outras Drogas. Brasília: MS; 2004.

2. Brasil. Politica Nacional sobre Drogas. Brasília: Conselho Nacional Antidrogas; 2005.

3. Brasil. Ministério da Saúde (MS). Reforma Psiquiátrica e política de saúde mental no Brasil. Brasília: MS; 2005.

4. Brasil. Portaria no 3.088, de 23 de dezembro de 2011. Institui a Rede de Atenção Psicossocial para pessoas com sofrimento ou transtorno mental e com necessidades decorrentes do uso de crack, álcool e outras drogas, no âmbito do Sistema Único de Saúde. Diário Oficial da União 2011; $24 \mathrm{dez}$.

5. Brasil. Tribunal de Contas da União (TCU). Sistema nacional de políticas públicas sobre drogas. Brasília: TCU; 2012.

6. Costa PHA, Mota DCB, Paiva FS, Ronzani TM. Desatando a trama das redes assistenciais sobre drogas: uma revisão narrativa da literatura. Cien Saude Colet 2015; 20(2):395-406.

7. Cordeiro LRO, Oliveira MS, Souza RC. Produção científica sobre os Centros de Atenção Psicossocial. Rev. esc. enferm. USP 2012; 46(1):119-123.

8. Lima FC, Schneider DR. Avaliação dos centros de atenção psicossocial: uma revisão integrativa da literatura nacional. Revista Caminhos 2013; 4(6):39-64.

9. Instituto Brasileiro de Geografia e Estatística (IBGE). 2016.Cidades@. [acessado 2016 Jul 15].Disponível em: http://www.cidades.ibge.gov.br/xtras/perfil.php?lan$\mathrm{g}=\& \operatorname{codmun}=313670 \&$ search $=$ minas-gerais $\mid$ juiz-defora.

10. Gomide M, Grossetti M. Rede social e desempenho de programas de saúde: uma proposta investigativa. Physis 2010; 20(3):873-893.

11. Bittencourt ONS, Neto FJK. Rede social no sistema de saúde: um estudo das relações interorganizacionais em unidades de serviços de HIV/AIDS. Rev. adm. contemp. 2009; 13(n. esp):87-104.

12. Blanchet K, James P. How to do (or not to do) ... a social network analysis in health systems research. Health Policy Plan. 2011; 27(5):438-446.

13. Smith KP, Christakis NA. Social Networks and Health. Annu. Rev. Sociol. 2008; 34:405-429.

14. Hawe P, Webster C, Shiell A. A glossary of terms for navigating the field of social network analysis. J Epidemiol Community Health 2004; 58(12):971-975.

15. Biernacki P, Waldford D. Snowball sampling: problems and techniques of chain referral sampling. Sociol Methods Res. 1981; 2:141-163.

16. R Development Core Team. R: A language and environment for statistical computing. Vienna: R Foundation for Statistical Computing; 2011.
17. Csardi G, Nepusz T. The igraph software package for complex network research. InterJournal Complex Systems 2006; 1695.

18. Amarante P. A clínica e a Reforma Psiquiátrica. In: Amarante P, organizador. Archivos de Saúde Mental e Atenção Psicossocial. Rio de Janeiro: NAU Editora; 2003. p. 45-66.

19. Costa PHA, Laport TJ, Mota DCB, Ronzani T M. A rede assistencial sobre drogas segundo seus próprios atores. Saúde Debate 2013; 37(n. esp.):110-121.

20. Eslabão AD, Coimbra VCC, Kantorski LP, Pinho LB, Santos EO. Rede de cuidado em saúde mental: visão dos coordenadores da estratégia saúde da família. Revista Gaúcha de Enfermagem 2017; 38(1):e60973.

21. Sala de Apoio à Gestão Estratégica (SAGE). 2016. [acessado 2017 Out 30]. Disponível em: http://sage.saude. gov.br/

22. Gonçalves VM, Abreu PS, Candiago RH, Saraiva SS, Lobato MI, Belmonte-de-Abreu PS. A falácia da adequação da cobertura dos Centros de Atenção Psicossocial no estado do Rio Grande do Sul. Rev. psiquiatr. Rio Gd. Sul 2010; 32:16-18.

23. Carvalho DBB, organizador. Mapeamento das instituições governamentais e não governamentais de atenção às questões relacionadas ao consumo de álcool e outras drogas no Brasil - 2006/2007. Brasília: Secretaria Nacional Antidrogas; 2007.

24. Santos MPG. Comunidades Terapêuticas no Brasil: contornos, funções e objetivos. Brasília: Instituto de Pesquisa Econômica Aplicada; 2014.

25. Observatório Crack, é Possível Vencer. 2016. [acessado 2016 Jul 15]. Diponível em: http://www.brasil.gov.br/ observatoriocrack/index.html

26. Vargas D, Duarte FAB. Enfermeiros dos centros de atenção psicossocial em álcool e drogas (Caps ad): a formação e a busca pelo conhecimento específico da área. Texto Contexto Enferm 2011; 20(1):119-126.

27. Larentis CP, Maggi A. Centros de Atenção Psicossocial Álcool e Drogas e a Psicologia. Aletheia 2012; 37(1):121-132.

28. Costa PHA, Paiva FS. Revisão de literatura das concepções dos profissionais de saúde sobre o uso de drogas: modelo biomédico, naturalizações e moralismos. Physis 2016; 26(3):1009-1031.

29. Bezerra E, Dimenstein M. Os CAPS e o trabalho em rede: tecendo o apoio matricial na atenção básica. Psicol. cienc. prof. 2008; 28(3):632-645.

30. Bernardi AB, Kanan LA. Características dos serviços públicos de saúde mental (Capsi, Capsad, Caps III) do estado de Santa Catarina. Saúde debate 2015; 39(107):1105-1116. 
31. Ribeiro JM, Moreira MR, Bastos FI, Inglez-Dias A, Fernandes FMB. Acesso aos serviços de atenção em álcool, crack e outras drogas - o caso do município do rio de Janeiro, Brasil. Cien Saude Colet 2016; 21(1):71-81.

32. Vasconcelos EM. Crise mundial, conjuntura política e social no Brasil, e os novos impasses teóricos na análise da reforma psiquiátrica no país. Cad. Bras. Saúde Mental 2012; 4(8):8-21.

33. Andrade TM. Reflexões sobre políticas de drogas no Brasil. Cien Saude Colet 2011; 16(12):4665-4674.

34. Rosenstock KIV, Neves MJ. Papel do enfermeiro da atenção básica de saúde na abordagem ao dependente de drogas em João Pessoa, PB, Brasil. Rev. Bras. Enferm. 2010; 63(4):581-586.

35. Paula ML, Jorge MSB, Vasconcelos MGF, Albuquerque RA. Assistência ao usuário de drogas na atenção primária à saúde. Psicologia em Estudo 2014; 19(2):223-233.

36. Campos RTO, Furtado JP. Entre a saúde coletiva e a saúde mental: um instrumental metodológico para avaliação da rede de Centros de Atenção Psicossocial (CAPS) do Sistema Único de Saúde. Cad Saude Publica 2006; 22(5):1053-1062.

37. Dimenstein MD, Liberato MTC. Desinstitucionalizar é ultrapassar fronteiras sanitárias: o desafio da intersetorialidade e do trabalho em rede. Cadernos Brasileiros de Saúde Mental 2009; 1(1):212-222.

Artigo apresentado em 21/12/2017

Aprovado em 06/03/2018

Versão final apresentada em 16/05/2018 
